\title{
The Effect of Adrenal Replacement Therapy on Rates of Fungal Colonization and Mortality in Critically Ill Patients Awaiting Liver Transplantation
}

\author{
Stephanie G. Yi ${ }^{1}$, Archana R. Sadhu ${ }^{2}$, Stephen L. Jones ${ }^{1}$, Krista Turner ${ }^{3}$, Howard Monsour ${ }^{4}$, \\ Kevin Donahue ${ }^{5}$, Xuefeng Xia ${ }^{6}$, A. Osama Gaber ${ }^{7}$, R. Mark Ghobrial ${ }^{7}$ and \\ Sherilyn Gordon Burroughs ${ }^{7}$
}

${ }^{1}$ Department of Surgery, The Methodist Hospital, Weill Cornell Medical College, Houston, TX; ${ }^{2}$ Division of Endocrinology, Department of Medicine, The Methodist Hospital, Houston, TX; ${ }^{3}$ Department of Surgery, University of New Mexico at Albuquerque, Albuquerque, NM; ${ }^{4}$ Hepatology and Transplant Medicine, Department of Medicine, The Methodist Hospital, Houston, TX; ${ }^{5}$ Department of Pharmacy, The Methodist Hospital, Houston, TX; ${ }^{6}$ The Methodist Hospital Research Institute, The Methodist Hospital, Houston, TX; ${ }^{7}$ Liver Transplantation and Hepatobiliary Surgery, Department of Surgery, The Methodist Hospital, Weill Cornell Medical College, Houston, TX

\begin{abstract}
Background: The effect of adrenal replacement therapy (ART) with hydrocortisone on critical endpoints such as infection and mortality in critically ill patients with cirrhosis remains unclear. We evaluated our indications for ART in patients with cirrhosis with clinical symptoms of adrenal insufficiency $(\mathrm{AI})$, and examined the rate of peri-transplant fungal colonization and mortality associated with ART.

Methods: Seventy-eight patients with cirrhosis admitted to our institution's surgical intensive care unit (ICU) over a 4year period met criteria for AI by vasopressor requirement and baseline cortisol levels. Outcomes included disposition at 90-days, fungal colonization, and fungal infection in the presence or absence of ART.

Results: In total, 56 patients received hydrocortisone $(\mathrm{HC}+)$ while 22 did not $(\mathrm{HC}-)$. The $\mathrm{HC}+$ and $\mathrm{HC}-$ groups had comparable median Model for End-stage Liver Disease (MELD) scores (26.5 vs. 25, respectively; $p=0.93$ ), median ICU lengths of stay ( 23 vs. 20 days, respectively; $p=0.54$ ) and median cortisol levels $(18 \mu \mathrm{g} / \mathrm{dL}$ for both, $p=0.87)$. Fungal cultures (FC) from blood, urine or bronchoalveolar lavage/sputum were positive for $44 \%$ of $\mathrm{HC}+$, and $40.9 \%$ of $\mathrm{HC}-(p=0.77)$ had mortality rates between $\mathrm{HC}+$ and $\mathrm{HC}-$ groups that were not significantly different $(60.7 \%$ vs. $50 \%$, respectively; $p=0.39 ; \alpha=0.05)$. The 90 -day outcomes for $\mathrm{HC}+$ vs. $\mathrm{HC}-(39.3 \%$ vs. $50 \%$ discharged, respectively; $p=0.39 ; \alpha=0.05)$ and those surviving to transplant $(17.9 \%$ vs. $36.4 \%$, respectively; $p=0.08 ; \alpha=0.05$ ) were not significantly different between the two groups.
\end{abstract}

Keywords: Sepsis; Hydrocortisone; Antifungal; Transplant. Abbreviations: ACTH, adrenocorticotropic hormone; AI, adrenal insufficiency; ART, adrenal replacement therapy; BAL, bronchoalveolar lavage; CVA, cerebrovascular accident; ESLD, end-stage liver disease; FC, fungal culture; HC, hydrocortisone; HDL, high-density lipoprotein; OLT, orthotopic liver transplantation; MAP, mean arterial pressure; NAFLD, non-alcoholic fatty liver disease. Received: 14 May 2013; Revised: 26 June 2013; Accepted: 28 June 2013

DOI of original article: http://dx.doi.org/10.14218/JCTH.2013.00007.

Correspondence to: Stephanie G. Yi, The Methodist Hospital, Department of Surgery, 6565 Fannin Street, SM 1661, Houston, TX 77030. Tel.: 713-441-8085, Fax: 713-790-2872, E-mail: sgyi@tmhs.org
Conclusion: In this small single-center series, we found that steroid administration for AI does not affect the rate of fungal colonization/infection or mortality. Further prospective studies are required to determine the utility of ART and factors affecting the rate of FC and mortality in these patients.

(C) 2013 The Second Affiliated Hospital of Chongqing Medical University. Published by XIA \& HE Publishing Ltd. All rights reserved.

\section{Introduction}

Symptoms suggestive of adrenal insufficiency (AI) are commonly observed in patients confined to the intensive care unit with cirrhosis awaiting orthotopic liver transplantation (OLT). ${ }^{1,2}$ Adrenal insufficiency is defined as a deficiency in either the production or action of glucocorticoids, and is often due to insufficient glucocorticoid activity in critical illness. ${ }^{3,4}$ The incidence and mortality associated with AI in patients without cirrhosis who are in septic shock have been reported to be as high as $61 \%{ }^{4}$ and $70 \%,{ }^{5}$ respectively. Use of traditional parameters studied in patients without liver disease suggests a $50-80 \%$ incidence of $\mathrm{AI}$ in patients with cirrhosis, ${ }^{6,7}$ with mortality rates ranging from 60 to $100 \% .7,8$ However, patients with end-stage liver disease (ESLD) in the absence of sepsis have clinical similarities to septic shock, such as high output cardiac failure, encephalopathy, and renal dysfunction. ${ }^{9}$ Concomitant infection or evolving sepsis may confound this clinical picture, and the condition may go undiagnosed unless the level of suspicion is high. Of particular concern is fungal infection, which may render the patient ineligible for transplantation, and/or increase the risk of peri-transplant mortality. ${ }^{10}$

Previous studies have been inconclusive regarding the survival benefit of adrenal replacement therapy (ART) in patients with sepsis without underlying liver disease, even with low-dose steroids ( $\leqslant 300 \mathrm{mg} /$ day of hydrocortisone or equivalent). ${ }^{11-14}$ The risk/benefit ratio of ART use in patients with late-stage cirrhosis is even less clear. Given the association of steroid administration with infection in the immunocompromised patient, ${ }^{10}$ the effects of ART on critical endpoints in patients with cirrhosis, such as peri-transplant 
infection (with potential loss of transplant eligibility) and peritransplant survival remain unknown. ${ }^{6,15,16}$

There is no current consensus by which to define AI in the context of chronic liver disease. Definitions for AI based on cortisol levels were obtained from critically ill patients without cirrhosis. ${ }^{17}$ The general consensus for AI in patients with sepsis without liver disease is a random cortisol level of $<15 \mu \mathrm{g} / \mathrm{dL}^{18}$ or a delta cortisol of $<9 \mu \mathrm{g} / \mathrm{dL}$ after $250 \mu \mathrm{g}$ cosyntropin stimulation test. ${ }^{19}$ Approximately $90 \%$ of circulating cortisol is bound to corticosteroid-binding globulin and albumin, and reduced levels of these proteins, as is typical in patients with cirrhosis, may lower total cortisol levels without affecting the free biologically active cortisol levels. ${ }^{20,21}$ Galbois et al. suggested that serum total cortisol levels overestimate the prevalence of adrenal insufficiency in patients with cirrhosis, given their decreased protein synthetic function. ${ }^{22}$

Due to depressed humoral and cell-mediated immunity, 23,24 high levels of circulating proinflammatory cytokines, ${ }^{25}$ and failure of gut integrity, ${ }^{26}$ patients with liver failure are predisposed to infection, particularly those caused by fungi. ${ }^{27,28}$ Post-OLT fungal infections result in poor outcomes with mortality rates reported to be as high as 70$92 \% .{ }^{29,30}$ Peri-transplant fungal infections have been shown to result in mortality rates as high as $72 \%, 10$ and could exclude patients from transplantation or could compromise post-transplant survival. Earlier studies performed on patients without cirrhosis including meta-analyses by Minneci et al. ${ }^{14}$ and Cronin et al., ${ }^{31}$ found an increased incidence of infection with low-dose ${ }^{5,12}$ to high-dose 32,33 ART; however, these findings could not be replicated in other studies reviewing lower-dose ART. ${ }^{11,14}$ Falcone et al. found that $58.3 \%$ of patients with liver disease and invasive aspergillosis infections had received concomitant steroid therapy. ${ }^{10}$

Because of the difficulty in arriving at an accurate biochemical diagnosis of AI, the uncertainty of therapeutic benefits of ART, and the increased risk of infection in liver disease, we sought to investigate the association between ART and the rate of positive fungal cultures and 90-day outcomes.

\section{Methods}

This retrospective study was conducted in the surgical intensive care unit (ICU) of a tertiary academic hospital. Charts of 257patients with cirrhosis requiring ICU monitoring over a 33-month period from 2008-2011 were reviewed.

The inclusion criteria were age $\geqslant 18$ years, presence of underlying cirrhosis diagnosed before or during ICU admission, current listing or evaluation for OLT in progress, and hemodynamic compromise as defined by any vasopressor requirement to maintain a mean arterial pressure (MAP) $>60 \mathrm{~mm} \mathrm{Hg}$. Patients with fulminant hepatic failure or previously on immunosuppression, including history of corticosteroid use or with prior transplantation, were excluded. All patients were co-managed by a multidisciplinary group of surgical intensivists, transplant surgeons, hepatologists, endocrinologists, and infectious disease clinicians. The study was approved by the institutional review board of the Methodist Hospital.

In total, 78 patients met the inclusion criteria, and were grouped according to whether ART in the form of intravenous hydrocortisone was administered $(\mathrm{HC}+)$ or not $(\mathrm{HC}-)$. The average dose of hydrocortisone or steroid dose equivalent was $204.28 \mathrm{mg} \pm 54.8$ per 24 hours. All received ART for at least 24 hours. The decision to administer ART was based on serum cortisol levels or evaluation of clinical symptoms suggestive of AI, such as vasopressor requirement, both at the discretion of the treating surgical intensivists. Serum cortisol levels were not obtained for patients who received etomidate. Prophylactic antifungal treatment in the form of an azole or echinocandin was initiated at the time of ART ( $\mathrm{HC}+$ ) or within 48 hours of obtaining cultures ( $\mathrm{HC}-$ ). Cultures were universally obtained in cases where there was clinical suspicion for fungemia, presence of other infection site, or declining patient status. ${ }^{34}$

Patients were compared by age, gender, liver disease etiology, and liver failure severity; the last was established at the time of ICU admission by the Model for End-stage Liver Disease (MELD) score. ${ }^{35}$ Fungal culture data and antifungal use were reviewed in all patients. Fungal cultures were obtained as described above. Positive cultures were further delineated by source, specifically bronchoalveolar lavage (BAL)/sputum, urine, blood, or mixed (from more than two positive sources). Hospitalization was monitored by ICU length of stay (LOS) in days. Patient outcomes were based on the status 90-days after ICU admission, and were defined as OLT (transplant), discharge to home or other non-acute care facility (discharged), and death (died). In addition to an available size and $\mathrm{ABO}$-appropriate matched graft, transplant eligibility at our center is determined by the following recipient criteria: sepsis-free, minimal ventilator settings and vasopressor utilization, and evidence of central nervous system responsiveness. ${ }^{36}$

Descriptive statistics are shown as mean \pm SD and median values with interquartile range. Unpaired Student $t$-tests $(\alpha=0.05)$ and Wilcoxon rank sum tests for nonparametric data were used for statistical analysis. Categorical data was analyzed using the $\chi^{2}$ test or Fisher's exact $t$-test if $\mathrm{n}<5$. Variables with $p<0.05$ were considered statistically significant. Statistical analysis was performed using STATA 12 software (StataCorp LP, College Station, TX, USA).

\section{Results}

\section{Patient demographics}

Of the 257 patients with cirrhosis admitted to the ICU, 78 patients met the criteria for inclusion; 56 (71.8\%) of these patients received hydrocortisone $(\mathrm{HC}+)$ and $22(28.2 \%)$ did not $(\mathrm{HC}-)$. The mean ages of the $\mathrm{HC}+$ and $\mathrm{HC}-$ cohorts were $55.2 \pm 9.1$ and $57.1 \pm 11.1$ years old, respectively $(p=0.48)$ (Table 1). The gender distribution was $51.8 \%$ men $(n=29)$ in the $\mathrm{HC}+$ group and $63.6 \%$ men $(\mathrm{n}=14)$ in the $\mathrm{HC}$ - group $(p=0.35)$. The median MELD was 26.5 (interquartile range $(\mathrm{IQR})=12.5))$ for $\mathrm{HC}+$ vs. $25(\mathrm{IQR}=10)$ for $\mathrm{HC}-,(p=0.93)$ ， and the median ICU LOS was 23 days $(\mathrm{IQR}=21.5)$ and 20 days $(\mathrm{IQR}=26)$, respectively $(p=0.54)$. Baseline cortisol levels were drawn for $47(83.9 \%) \mathrm{HC}+$ patients, and 14 $(63.6 \%) \mathrm{HC}-$ patients prior to starting ART. The median cortisol level was $18 \mu \mathrm{g} / \mathrm{dL}(\mathrm{IQR}=13)$ for the $\mathrm{HC}+$ group, and $18 \mu \mathrm{g} / \mathrm{dL}$ (IQR $=15$ for the $\mathrm{HC}-$ group $(p=0.87)$. Etomidate was given to 13 patients $(23.2 \%)$ in the $\mathrm{HC}+$ group, and 6 patients $(27.3 \%)$ in the $\mathrm{HC}-$ group. The etiology of liver disease for the HC+ group was as follows: $41.1 \%(n=23)$ alcoholic cirrhosis, 33.9\% $(n=19)$ viral hepatitis, and $25 \%$ $(n=14)$ "other etiologies", which comprised of cryptogenic 
Table 1. Patient Demographics

\begin{tabular}{llll}
\hline & $\mathrm{HC}+(\mathrm{n}=56)$ & $\mathrm{HC}-(\mathrm{n}=22)$ & $p(<0.05, \alpha=0.05)$ \\
\hline $\begin{array}{l}\text { Age, years } \\
\text { Gender, }\end{array}$ & $55.2 \pm 9.1$ & $57.1 \pm 11.1$ & 0.47 \\
Male & & & 0.47 \\
Female & $51.8 \%(\mathrm{n}=29)$ & $63.6 \%(\mathrm{n}=14)$ & \\
Etiology & $48.2 \%(\mathrm{n}=27)$ & $36.4 \%(\mathrm{n}=8)$ & 0.006 \\
Alcohol & & & 0.09 \\
Viral hepatitis & $41.1 \%(\mathrm{n}=23)$ & $9.1 \%(\mathrm{n}=2)$ & 0.32 \\
Other & $33.9 \%(\mathrm{n}=19)$ & $54.5 \%(\mathrm{n}=12)$ & \\
$\quad$ Cryptogenic & $25 \%(\mathrm{n}=14)$ & $36.3 \%(\mathrm{n}=8)$ & \\
$\quad \mathrm{n}=1$ & $\mathrm{n}=3$ & $\mathrm{n}=3$ & 0.93 \\
$\quad \mathrm{NAFLD}$ & $\mathrm{n}=0$ & $\mathrm{n}=1$ & 0.87 \\
$\quad \mathrm{n}=6$ & $\mathrm{n}=1$ & 0.54 \\
MELD, median & $\mathrm{n}=7$ & $25(\mathrm{IQR}=22-32)$ & \\
Cortisol level, $\mu \mathrm{g} / \mathrm{dL} ;$ median & $26.5(\mathrm{IQR}=20-32.5)$ & $18(\mathrm{IQR}=15, \mathrm{n}=14)$ & \\
ICU LOS, days median & $18(\mathrm{IQR}=13, \mathrm{n}=47)$ & $20(\mathrm{IQR}=10-36)$ & \\
\hline
\end{tabular}

Comparison of patients who received low-dose hydrocortisone $(\mathrm{HC}+)$ and patients who did not (HC-). ICU LOS = Intensive care unit length of stay (in days), IQR = interquartile range; MELD = Model for End Stage Liver Disease scores, NAFLD = non-alcoholic fatty liver disease. 'Other' etiologies included cryptogenic, cholestatic liver disease, NAFLD, and congenital. 'Congenital' includes polycystic liver disease, biliary atresia, $\alpha 1$ antitrypsin deficiency, and hemochromatosis.

$(n=1)$, non-alcoholic fatty liver disease (NAFLD; $n=6)$, and congenital ( $\alpha 1$ antitrypsin deficiency, biliary atresia, and hemochromatosis) $(n=7)$. The etiologies of liver disease in the HC- group were $9.1 \%(n=2)$ alcoholic cirrhosis $(p=0.006), 54.5 \%(n=12)$ viral hepatitis $(p=0.09)$, and other etiologies $36.3 \%(n=8)(p=0.32)$, which included cryptogenic $(n=3)$, cholestatic $(n=3), \operatorname{NAFLD}(n=1)$, and congenital (polycystic) $(n=1)$.

\section{Culture results}

Fungal cultures (FC) were obtained for all patients in each group; however, only $46.4 \%(n=26)$ of $\mathrm{HC}+$ patients and $27.2 \%(n=6)$ of $\mathrm{HC}-$ patients had indications for blood FC $(p=0.12)$. Positive FC of any type was found in $44.6 \%(n=25)$ of the $\mathrm{HC}+$ group, and $40.9 \%(n=9)$ of the $\mathrm{HC}$ - group $(p=0.77)$. Overall, there was no statistically significant relationship between the distribution of positive fungal cultures and steroid administration $(p=0.812)$. Of the $\mathrm{HC}+$ positive FC, $24 \%(n=6)$ were from BAL/sputum, 36\% $(n=9)$ from urine, $12 \%(n=3)$ from blood and $28 \%(n=7)$ from more than two sources (Fig. 2). For the HC- patients the sources were: $22.2 \%(n=2)$ BAL/sputum ( $p>0.99), 22.2 \%(n=2)$ urine $(p=0.38), 11.1 \%(n=1)$ blood $(p>0.99)$, and $44.4 \%$ $(\mathrm{n}=4)$ mixed $(p=0.31)$ (Fig. 1$)$.

\section{Patient disposition}

The 90-day outcomes were evaluated in both groups. Of the $\mathrm{HC}+$ cohort, $17.9 \%(\mathrm{n}=10)$ survived to transplant, and one patient died in the immediate postoperative period from antibody-mediated rejection. In the HC-group, 36\% $(n=8)$ survived to transplant ( $p=0.08$ ) (Fig. 2 ), and one patient died in the immediate postoperative period from a cerebrovascular accident (Fig. 3). Of the patients with high suspicion of fungemia, $30 \%(1 / 3)$ in the HC+ group died, compared with
$20 \%(1 / 5)$ in the $\mathrm{HC}$ - cohort ( $\mathrm{p}>0.99)$. Overall, in the $\mathrm{HC}+$ group, $60.7 \%(n=34)$ died from a sepsis-related cause, while $39.3 \%(n=22)$ were discharged to home or a facility. Within the HC- cohort, $50 \%(n=11)$ died within the 90-day followup period $(p=0.39)$ and $50 \%(n=11)$ were discharged $(p=0.39)$. Of the patients in the $\mathrm{HC}+$ cohort who survived, $45.5 \%$ had alcoholic cirrhosis $(n=11), 31.8 \%$ had viral hepatitis $(n=7), 9.1 \%$ had NAFLD $(n=9)$, and $13.6 \%$ had congenital cirrhosis $(n=3)$, while the numbers in the HCcohort were $18.1 \%$ alcoholic cirrhosis $(n=2), 36.4 \%$ viral hepatitis $(n=4), 9.1 \%$ cryptogenic cirrhosis $(n=1), 18.2 \%$ cholestatic cirrhosis $(n=2), 9.1 \%$ NAFLD cirrhosis $(n=1)$, and $9.1 \%$ congenital cirrhosis $(n=1)$.

\section{Discussion}

Critically ill patients with cirrhosis are at high risk for infection, particularly fungal infections, ${ }^{23,37}$ which may compromise OLT candidacy. In this small retrospective series, we found that low-dose ART did not have a statistically significant effect on the rate or distribution of peri-transplant fungal colonization/infection or ability to maintain candidacy for OLT. Although factors such as number and duration of vasopressors, or positivity of bacterial cultures were not included in this analysis, the two patient groups were equivalent with regards to disease severity (as measured by MELD), age, and distribution of causes of cirrhosis. There was a significantly higher representation of patients with alcoholic cirrhosis in the $\mathrm{HC}+$ group; however, given the small cohort size, it is unclear as to how the etiology of cirrhosis may have influenced the effects of ART in our study population. Evaluation of fungal culture data showed no significant difference in colonization, infection rate, or site distribution between the two groups. Patient outcome was also similar between the two cohorts. Despite a higher raw percentage of $\mathrm{HC}+$ patients expiring $(60.7 \% \mathrm{HC}+$ vs. $50 \% \mathrm{HC}-)$, and a 
Yi S.G. et al: Outcomes of steroid administration in patients with cirrhosis

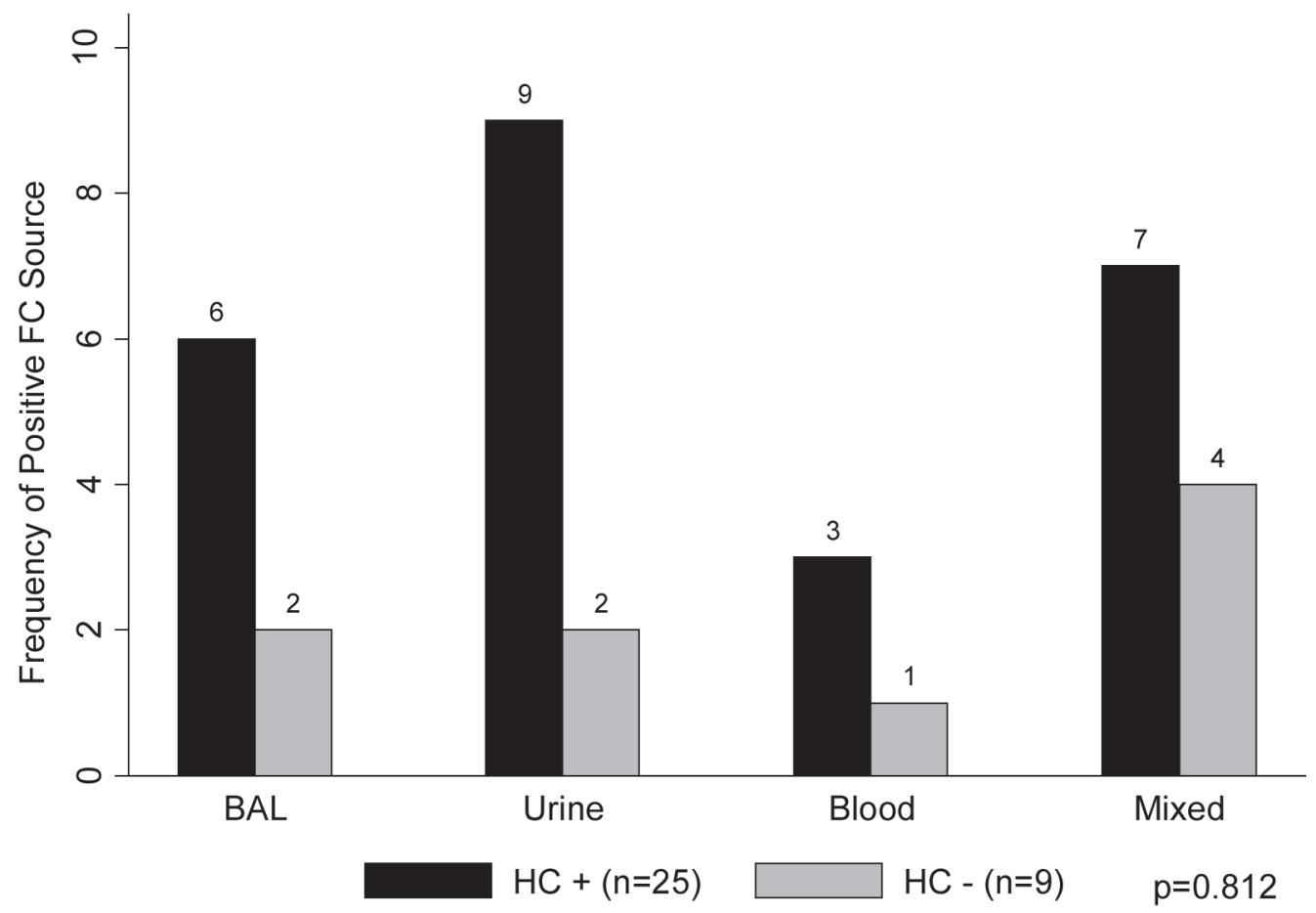

Fig. 1. Sources of positive fungal cultures. Sources of fungal cultures (FC). Culture location was based on broncheoalveolar lavage (BAL) or sputum, urine, blood or $\geqslant 2$ sources (mixed).

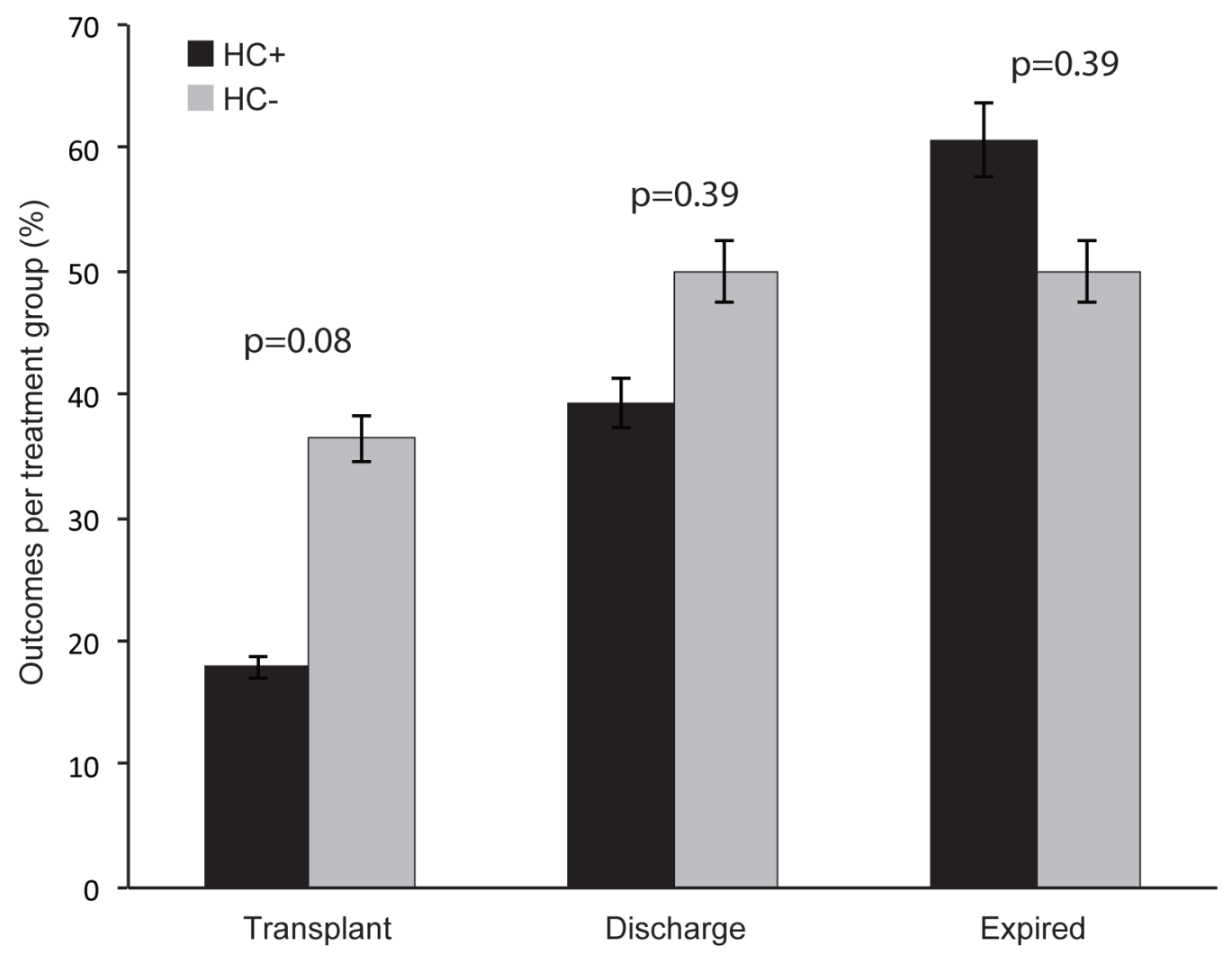

Fig. 2. Patient disposition per treatment group at $\mathbf{9 0}$ days. Patient outcomes at 90 days after admission to the intensive care unit. Transplant, OLT; discharge, release to home or facility; and expired, death from sepsis-related causes. 


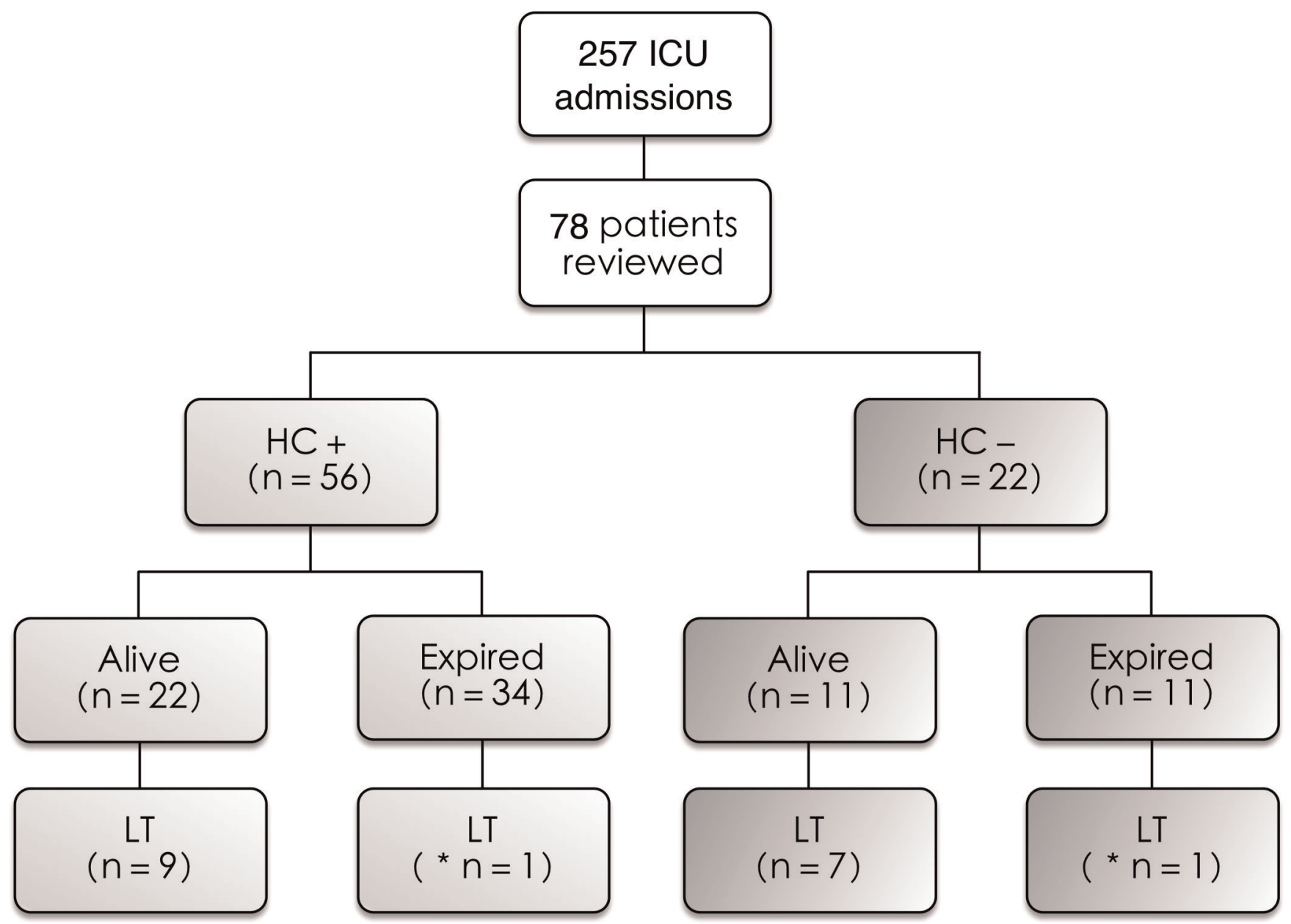

Fig. 3. Flowchart of patient distribution and 90day outcomes. All patients were diagnosed with cirrhosis prior to admission. Patients were reviewed based on eligibility of study criteria as described in the methods section. Alive, alive at 90-days post-ICU admission and discharged to home or non-acute care facility; ICU, intensive care unit. $\mathrm{HC}+$, received hydrocortisone, $\mathrm{HC}-$, did not receive hydrocortisone, $\mathrm{LT}$, liver transplantation. *Patients who received transplants, but died within the postoperative period were considered deaths. Causes of mortality: cerebrovascular accident and hyperacute cell-mediated rejection.

lower percentage of $\mathrm{HC}+$ patients maintaining eligibility for transplant $(17.9 \% \mathrm{HC}+$ vs. $36.4 \% \mathrm{HC}-$ ), no statistical significance could be found between the two groups. Because this study was insufficiently powered, we cannot conclude that there is a relationship between survival to OLT and ART use. However, it is interesting to note that steroids for AI may not confer a survival benefit in critically ill patients with liver failure.

The role of ART in AI has been controversial, ${ }^{1-14}$ and although several studies reported a decrease in vasopressor requirement, ${ }^{6,15,16,32}$ there has not been consistent evidence demonstrating an improvement in mortality $6,15,16,32$ in patients without liver disease. There is likewise no consensus on the ideal duration of ART in patients with liver disease, with studies utilizing time to shock resolution ${ }^{15,16}$ unlike the 7days proposed by Annane et al. ${ }^{11}$ for patients without cirrhosis. Additionally, the duration of therapy may confer an increased risk of adverse effects, as studies have reported increases in gastrointestinal bleeding ${ }^{15}$ and superinfection. ${ }^{16}$ Several studies have suggested that a reduction in vasopressor treatment duration may increase the chances of becoming eligible to obtain a transplant; ${ }^{6,32}$ however, this was not an outcome evaluated in our study.

Although the exact mechanism for AI in cirrhosis is not well defined, studies suggest that the pathology for AI in cirrhosis may be different than in patients without chronic liver disease. ${ }^{38}$ Described as "hepatoadrenal syndrome,", ${ }^{6} \mathrm{AI}$ in liver disease is proposed to be a result of increased circulating endotoxins, which exacerbate the already depressed synthetic function of the liver by elevating proinflammatory cytokine levels, and further decreasing production of steroidal substrates. ${ }^{39}$ The association between depressed high-density lipoprotein (HDL) levels ${ }^{40}$ and the inhibitory affect of cytokines on cortisol production ${ }^{41}$ suggest impaired cortisol synthesis in these patients. In patients with cirrhosis without sepsis or hemodynamic instability, $60 \%$ have been found to have an abnormally low response to adrenocorticotropic hormone (ACTH) stimulation test, ${ }^{42}$ raising the possibility that AI may be a pre-existing condition exacerbated by critical illness or may be the result of a poorly measured assessment of adrenal function in this population. 22,43 
The difficulty of making a biochemical diagnosis, and lack of current consensus for a definition of AI in the context of chronic liver disease suggests a need to evaluate alternative assays specific to this patient population. Standard assays measure serum total cortisol concentration, which is composed of two fractions: serum-free cortisol, which is physiologically active, and protein-bound cortisol, which is inactive. Serum-free cortisol levels were not evaluated in the current study, and are not readily available. Additionally, decisions for administering ART in this study, as well as in previous studies, ${ }^{6,32}$ were often based upon clinical judgment, such as vasopressor requirement and response to vasopressors. Further analysis utilizing cortisol-binding globulin and free serum cortisol levels will be necessary to better define the effect of AI on the outcomes of critically ill patients with cirrhosis, as well as to evaluate the role of clinical judgment versus predetermined parameters in determining treatment.

There are several limitations to our retrospective study. First, our small sample size increased the likelihood of a type II error. We excluded $70 \%$ of our patients because of prior steroid use or lack of vasopressor requirement. Secondly, because it was a retrospective study, we could not control for the variability in indications for fungal culture analysis and variation in antifungal prophylaxis. Additionally, not all patients had cortisol levels obtained, as treatment was based on the clinical judgment of the intensivists. These limitations may have introduced bias within the sample. The uniform use of ACTH stimulation tests would have provided additional information for AI, although etomidate use was proportionally similar between both groups. Lastly, the limitation inherent to any study of this nature is the availability of donor organs, which cannot be controlled, but affects patient survival to OLT.

As the number of patients with cirrhosis listed for transplantation frequently exceeds graft availability, the numbers of critically ill liver patients will increase. Their hyperdynamic physiologic profile, consisting of a high cardiac output with low systemic venous resistance, may compound a picture that classically suggests AI in a patient without cirrhosis. Despite a lack of definitive diagnostics or evidence for a survival benefit with ART, these patients are often administered ART. In this small retrospective study, we have demonstrated that ART likely has no statistically significant affect on peri-transplant fungal colonization and infection, and furthermore may not confer survival in patients with cirrhosis with clinical features of AI. A novel aspect to our study is our analysis of 90-day survival outcomes, which extends our surveillance beyond the index ICU admission. There also appeared to be a higher percentage of patients going to transplant in the HC- group and, although this observation nearly reached statistical significance, this trend is multi-factorial and dependent on graft availability. Future studies incorporating prospective analysis of cortisolbinding globulin and free serum cortisol levels in larger cohorts of patients with cirrhosis will be required to confirm these results.

\section{Author contributions}

Study design (SGY, KD, SGB), data analysis (SGY, SLJ, SGB), manuscript writing (SGY, ARS, SGB), critical revision (SGY, ARS,KT, HM, SLJ, XX, AOG, RMG, SGB)

\section{Conflict of interest}

None

\section{References}

[1] Clemmesen O, Ott P, Larsen FS. Splanchnic metabolism in acute liver failure and sepsis. Curr Opin Crit Care 2004;10:152-155.

[2] Blendis L, Wong F. The hyperdynamic circulation in cirrhosis: An overview. Pharmacol Ther 2001;89:221-231.

[3] Marik PE, Zaloga GP. Adrenal insufficiency during septic shock. Crit Care Med 2003; $31: 141-145$

[4] Marik PE, Pastores SM, Annane D, Meduri GU, Sprung CL, Arlt W, et al. American College of Critical Care Medicine. Recommendations for the diagnosis and management of corticosteroid insufficiency in critically ill adult patients: Consensus statements from an international task force by the american college of critical care medicine. Crit Care Med 2008;36:19371949.

[5] Annane D, Sebille V, Charpentier C, Bollaert PE, Francois B, Korach JM, et al. Effect of treatment with low doses of hydrocortisone and fludrocortisone on mortality in patients with septic shock. JAMA 2002;288:862-871.

[6] Marik PE, Gayowski T, Starzl TE, Hepatic Cortisol Research and Adrenal Pathophysiology Study Group. The hepatoadrenal syndrome: A common yet unrecognized clinical condition. Crit Care Med 2005;33:1254-1259.

[7] Tsai MH, Peng YS, Chen YC, Liu NJ, Ho YP, Fang JT, et al. Adrenal insufficiency in patients with cirrhosis, severe sepsis and septic shock. Hepatology 2006 43:673-681.

[8] Moreau R, Hadengue A, Soupison T, Kirstetter P, Mamzer MF, Vanjak D, et al. Septic shock in patients with cirrhosis: Hemodynamic and metabolic characteristics and intensive care unit outcome. Crit Care Med 1992;20: 746-750.

[9] Thierry S, Giroux Leprieur E, Lecuyer L, Brocas E, Van de Louw A. Echocardiographic features, mortality, and adrenal function in patients with cirrhosis and septic shock. Acta Anaesthesiol Scand 2008;52:45-51.

[10] Falcone M, Massetti AP, Russo A, Vullo V, Venditti M. Invasive aspergillosis in patients with liver disease. Med Mycol 2011;49:406-413.

[11] Annane D, Bellissant E, Bollaert PE, Briegel J, Keh D, Kupfer Y. Corticosteroids for treating severe sepsis and septic shock. Cochrane Database Syst Rev 2004; CD002243.

[12] Sprung CL, Annane D, Keh D, Moreno R, Singer M, Freivogel K, et al. Hydrocortisone therapy for patients with septic shock. N Engl J Med 2008; 358:111-124.

[13] Annane D, Bellissant E, Bollaert PE, Briegel J, Confalonieri M, De Gaudio R, et al. Corticosteroids in the treatment of severe sepsis and septic shock in adults: A systematic review. JAMA 2009;301:2362-2375.

[14] Minneci PC, Deans KJ, Eichacker PQ, Natanson C. The effects of steroids during sepsis depend on dose and severity of illness: An updated metaanalysis. Clin Microbiol Infect 2009;15:308-318.

[15] Arabi YM, Aljumah A, Dabbagh O, Tamim HM, Rishu AH, Al-Abdulkareem A, et al. Low-dose hydrocortisone in patients with cirrhosis and septic shock: $A$ randomized controlled trial. CMAJ 2010;182:1971-1977.

[16] Fernandez J, Escorsell A, Zabalza M, Felipe V, Navasa M, Mas A, et al. Adrenal insufficiency in patients with cirrhosis and septic shock: Effect of treatment with hydrocortisone on survival. Hepatology 2006;44:1288-1295.

[17] Oelkers W. Adrenal insufficiency. N Engl J Med 1996;335:1206-1212.

[18] Cooper MS, Stewart PM. Corticosteroid insufficiency in acutely ill patients. N Engl J Med 2003;348:727-734.

[19] Marik PE, Pastores SM, Annane D, Meduri GU, Sprung CL, Arlt W, et al. Recommendations for the diagnosis and management of corticosteroid insufficiency in critically ill adult patients: Consensus statements from an international task force by the american college of critical care medicine. Crit Care Med 2008;36:1937-1949.

[20] Hamrahian AH, Oseni TS, Arafah BM. Measurements of serum free cortisol in critically ill patients. N Engl J Med 2004;350:1629-1638.

[21] McDonald JA, Handelsman DJ, Dilworth P, Conway AJ, McCaughan GW. Hypothalamic-pituitary adrenal function in end-stage non-alcoholic liver disease. J Gastroenterol Hepatol 1993;8:247-253.

[22] Galbois A, Rudler M, Massard J, Fulla Y, Bennani A, Bonnefont-Rousselot D, et al. Assessment of adrenal function in cirrhotic patients: Salivary cortisol should be preferred. J Hepatol 2010;52:839-845

[23] Cheruvattath $R$, Balan V. Infections in patients with end-stage liver disease. J Clin Gastroenterol 2007;41:403-411.

[24] Campbell AC, Dronfield MW, Toghill PJ, Reeves WG. Neutrophil function in chronic liver disease. Clin Exp Immunol 1981;45:81-89.

[25] Byl B, Roucloux I, Crusiaux A, Dupont E, Deviere J. Tumor necrosis factor alpha and interleukin 6 plasma levels in infected cirrhotic patients. Gastroenterology 1993;104:1492-1497. 
[26] Bonnel AR, Bunchorntavakul C, Reddy KR. Immune dysfunction and infections in patients with cirrhosis. Clin Gastroenterol Hepatol 2011;9: 727-738.

[27] Prodanovic H, Cracco C, Massard J, Barrau OLT C, Thabut D, Duguet A, et al. Invasive pulmonary aspergillosis in patients with decompensated cirrhosis: Case series. BMC Gastroenterol 2007;7(1):2.

[28] Bajaj JS, O'Leary JG, Reddy KR, Wong F, Olson JC, Subramanian RM, et al. Second infections independently increase mortality in hospitalized cirrhotic patients: the North American consortium for the study of end-stage liver disease (NACSELD) experience. Hepatology 2012;56:2328-2335.

[29] Husain S, Tollemar J, Dominguez EA, Baumgarten K, Humar A, Paterson DL, et al. Changes in the spectrum and risk factors for invasive candidiasis in liver transplant recipients: Prospective, multicenter, case-controlled study. Transplantation 2003;75:2023-2029.

[30] Singh N, Avery RK, Munoz P, Pruett TL, Alexander B, Jacobs R, et al. Trends in risk profiles for and mortality associated with invasive aspergillosis among liver transplant recipients. Clin Infect Dis 2003;36:46-52.

[31] Cronin L, Cook DJ, Carlet J, Heyland DK, King D, Lansang MA, et al. Corticosteroid treatment for sepsis: A critical appraisal and meta-analysis of the literature. Crit Care Med 1995;23:1430-1439.

[32] Harry R, Auzinger G, Wendon J. The effects of supraphysiological doses of corticosteroids in hypotensive liver failure. Liver Int 2003;23:71-77.

[33] Bone RC, Fisher C], Jr, Clemmer TP, Slotman G], Metz CA, Balk RA. A controlled clinical trial of high-dose methylprednisolone in the treatment of severe sepsis and septic shock. N Engl J Med 1987;317:653-658.

[34] Dellinger RP, Levy MM, Carlet JM, Bion J, Parker MM, Jaeschke R, et al. Surviving sepsis campaign: International guidelines for management of severe sepsis and septic shock: 2008. Intensive Care Med 2008; 34: 17-60.
[35] United Network for Organ Sharing. Questionas \& Answers for transplant candidates about liver allocation policy. http://www.unos.org/docs/ Liver_patient.pdf. Accessed August 2011.

[36] Gordon Burroughs S, Gaber OA, Ghobrial RM. Liver Transplantation Protocol The Methodist Hospital, 2008.

[37] Plessier A, Denninger MH, Consigny Y, Pessione F, Francoz C, Durand F, et al. Coagulation disorders in patients with cirrhosis and severe sepsis. Liver Int $2003 ; 23: 440-448$.

[38] Albillos A, de la Hera A, Gonzalez M, Moya JL, Calleja JL, Monserrat J, et al. Increased lipopolysaccharide binding protein in cirrhotic patients with marked immune and hemodynamic derangement. Hepatology 2003;37: 208-217.

[39] O'Beirne J, Holmes M, Agarwal B, Bouloux P, Shaw S, Patch D, et al. Adrenal insufficiency in liver disease - what is the evidence? J Hepatol 2007;47:418423.

[40] Etogo-Asse FE, Vincent RP, Hughes SA, Auzinger G, Le Roux CW, Wendon J, et al. High density lipoprotein in patients with liver failure; relation to sepsis, adrenal function and outcome of illness. Liver Int 2012;32:128-136.

[41] Jaattela M, Ilvesmaki V, Voutilainen R, Stenman UH, Saksela E. Tumor necrosis factor as a potent inhibitor of adrenocorticotropin-induced cortisol production and steroidogenic P450 enzyme gene expression in cultured human fetal adrenal cells. Endocrinology 1991;128:623-629.

[42] Fede G, Spadaro L, Tomaselli T, Privitera G, Piro S, Rabuazzo AM, et al. Assessment of adrenocortical reserve in stable patients with cirrhosis. J Hepatol 2011;54:243-250.

[43] Thevenot T, Borot S, Remy-Martin A, Sapin R, Cervoni JP, Richou C, et al. Assessment of adrenal function in cirrhotic patients using concentration of serum-free and salivary cortisol. Liver Int 2011;31:425-433. 